\title{
Naturally occuring canine cancers: powerful models for stimulating pharmacogenomic advancement in
} human medicine

\section{"...research into canine cancer may offer a wealth of insight into driver mechanisms of human carcinogenesis and provide a more representative model for testing new therapies."}

\section{KEYWORDS: cancer " canine model $\|$ chemotherapy $\approx$ clinical trials}

An estimated 1.6 million new cases of cancer were diagnosed in the USA in 2012 [101]. The pharmaceutical industry is working furiously to develop new efficacious chemotherapeutics; however, the vast majority of compounds that show anticancer activity in preclinical studies fail during subsequent human clinical trials, hindering progress in patient care and further increasing costs for drug development [1-3]. Modern cancer research places a heavy emphasis on murine models for investigating cancer etiology and for driving the development of new therapies. Mice represent excellent models for studying cancer due to their short lifespans, ease of maintenance and opportunities for genetic manipulation [4]. While their attributes have led to numerous fundamental advances in identifying novel therapies, several important limitations exist. Murine models of cancer are generally induced by genetic engineering, or by subcutaneous xenografts. The limitations and advantages of various methods of inducing neoplasms in mice are well reviewed elsewhere [4,5]. Induced murine neoplasms are developed in a short period of time and they lack heterogeneity in the tumor cell population, the microenvironment and the stroma, all of which are inconsistent with most human cancers. Furthermore, human cancers typically display increased genomic instability compared with their induced murine counterparts, which limits their utility as tools for pharmacogenomics [6]. Many of these limitations may be addressed by using the domestic dog as a complementary model system. Canines share our environment and develop many age-related diseases with similar pathologies to humans. Perhaps most importantly, dogs exhibit a wide variety of spontaneous cancers that share extensive clinicopathologic features with those of human patients, offering a unique opportunity for comparative analysis of naturally occurring cancers towards advancing treatment strategies in both species.

"...the vast majority of compounds that show anticancer activity in preclinical studies fail during subsequent human clinical trials..."

Why canines make promising models of human cancer

A survey conducted from 2011-2012 indicates that approximately 78 million domestic dogs currently reside in the USA [102]. The domestic dog suffers from more than 450 spontaneous diseases, approximately 360 of which have a human analog [7]. Cancer is the leading cause of death in several of the most popular dog breeds, such as golden retrievers, Labrador retrievers and boxers, which succumb to cancer with frequencies of 50 , 34 and $44 \%$, respectively [8]. When evaluated by breed, there are clear differences in predispositions for a variety of cancers. In turn, several canine cancers exhibit breed-associated profiles of tumor-associated genomic aberrations, including lymphoma, osteosarcoma (OS) and intracranial malignancies. Moreover, comparative studies have revealed conserved genomic signatures in canine and human counterparts of the same cancer [9-12]. Based on the large population of dogs that develop cancer and the clinical and pathological similarity between many dog and human cancers, research into canine cancer may offer a wealth of insight into driver mechanisms of human carcinogenesis and provide a more representative model for testing new therapies.

\section{Canine pharmacogenomics}

The availability of a high-quality canine genome sequence assembly [13] has opened the door for identifying key drivers of disease that may also

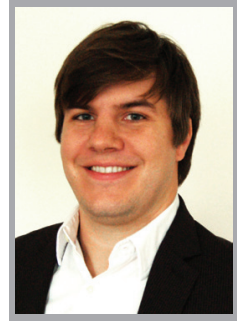

Daniel M Rotroff

Bioinformatics Research Center, Department of Statistics, Bioinformatics Research Center, North Carolina State University, Raleigh, NC 27695, USA

Rachael Thomas Department of Molecular Biomedical Sciences, North Carolina State University, Raleigh, NC, USA and Center for Comparative Medicine \& Translational Research, North Carolina State University, Raleigh, NC, USA

\section{Matthew Breen}

Department of Molecular Biomedical Sciences, North Carolina State University, Raleigh, NC, USA and Center for Comparative Medicine \& Translational Research, North Carolin State University, Raleigh, NC, USA and Cancer Genetics Program, UNC Lineberger Comprehensive Cancer Center, Chapel Hill, NC, USA

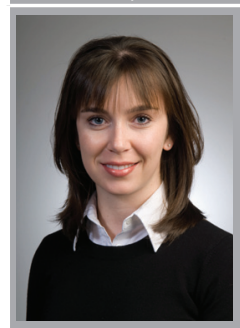

Alison A Motsinger-Reif Author for correspondence: Bioinformatics Research Center, Department of Statistics, Bioinformatics Research Center, North Carolina State University, Raleigh, NC 27695, USA motsinger@stat.ncsu.edu

\section{Future $\because \%$} Medicine part of 
impact human patients. In an effort to identify putative drivers of OS, Angstadt et al. combined bioinformatics and experimental methods to conduct a genome-wide assessment of tumorassociated DNA copy number aberrations in spontaneous canine and human OS [10]. OS in both species exhibits extensive genomic instability and a similar pathophysiological profile. Critically, however, human OS represents $<1000$ new diagnoses per year in the USA compared with $>10,000 /$ year in canines, thus providing tremendous sources of clinical materials [10]. Comparative analysis of recurrent copy number aberrations in human and canine OS revealed discrete gene sets that were common to both species, disrupting fundamental cellular functions such as osteoblast fate commitment, regulation of organ growth, telomerase activity and homophilic cell adhesion, among others [10]. These results shed new light on gene candidates that may play a role in OS development and progression in both species [10], reinforcing the enormous potential for advancement through cross-species approaches.

\section{Clinical potential of canines}

In addition to using tumor-bearing canines as preclinical models, methods for incorporating dogs into the existing clinical testing framework have been proposed, and a logical integrated approach has been well described by Paoloni and Khanna [6]. Since canine clinical management strategies utilize similar diagnostic and therapeutic modalities to those in human medicine, the diagnostic nomenclature of canine cancers broadly follows that of their human counterparts. Canine chemotherapy protocols focus on more palliative approaches and are therefore prescribed at lower doses than the equivalent human protocols [6]. For example, the standard protocol for canine B-cell non-Hodgkin's lymphoma (NHL) is cyclophosphamide, doxorubicin, vincristine and prednisone (CHOP), which, until recently, was also the first-line treatment for humans with B-cell NHL. In human patients, the recent addition of the monoclonal antibody rituximab to the $\mathrm{CHOP}$ regimen (i.e., R-CHOP) has become the first-line treatment for humans with B-cell NHL.

\section{"The domestic dog suffers from more than 450 spontaneous diseases, approximately 360 of which have a human analog."}

In the search for targeted lymphoma and autoimmune-related therapies, activation of the $\mathrm{B}$-cell antigen receptor signaling pathway has become a high priority since being identified as critical for the survival of B-cell NHL cells [14]. Btk represents a key component for B-cell antigen receptor activation, making the recent discovery of ibrutinib, the small-molecule irreversible inhibitor of Btk, a promising advance in the search for new and effective therapies for NHL $[14,15]$. In a recent study by Honigberg et al., murine models were used to demonstrate ibrutinib's efficacy for treating lupus and arthritis, and dogs with spontaneous B-cell NHL were enrolled in a trial to test whether ibrutinib would affect disease progression [15]. At the time the study was published, eight dogs had been treated, with three partial responses (one dog with a 77\% reduction in tumor burden) and three incidences of stable disease [15]. Upon these findings, a human clinical trial was promptly conducted on patients with refractory and relapsed B-cell NHL. Promising results were achieved, with a complete response in $16 \%$ of patients, while $60 \%$ of patients reached the objective response rate, and the median progression-free survival at the end of the trial for all patients was 13.6 months [16]. The US FDA has granted ibrutinib three 'breakthrough' designations and it is currently pending FDA approval. Hopes are high that it will see approval by the end of the year.

\section{“...companion animal studies are subject to strict regulatory oversight that is highly comparable to that enforced for human patients."}

\section{Ethics of the canine model}

While the use of companion animals has enormous potential for comparative medicine, it is important to recognize that these canine patients are presenting with naturally occurring disorders and are receiving medical care for their own well-being with informed owner consent. Any time that research is performed in a clinical setting (rather than a laboratory setting), there are a number of additional factors that must be considered. These include the patient environment, owners of animals and the perspective of the general public.

Many of the ethical concerns regarding involving companion animals in clinical research studies are shared with basic principles of human subjects, including autonomy (informed consent, in this case of the owners) and non-malfeasance (an absence of any sort of fraud or harmful deceit, as well as avoiding harm to the animal). Research conducted 
in this setting requires rigorous protection of confidential patient/owner data and must be performed to a high ethical standard. As such, companion animal studies are subject to strict regulatory oversight that is highly comparable to that enforced for human patients. These principles will inevitably impact the design of canine studies, but are also critical for assuaging concerns.

\section{Conclusion}

The murine model will continue to be an indispensable resource for cancer research and is deserving of enormous credit for the advancements in cancer therapy over previous decades. We believe that the large numbers of available canine patients with spontaneous cancers can offer an unequalled opportunity for stimulating advancement in the clinical management of human and companion animal cancers. We propose that, for applicable diseases, the canine model should be integrated into existing preclinical and clinical frameworks (including pharmacogenomics studies) in a way that not only benefits human drug development and disease understanding, but also improves the quality of treatment for our four-legged friends. Although we have focused primarily on cancer, it should also be noted that dogs are successfully used to study and develop treatments for many other diseases and disorders, such as wound healing, blindness and other vision disorders, heart disease, epilepsy, autoimmune diseases and narcolepsy [17,18]. It is our hope that with continued integration in these areas, we can expedite both the identification of druggable targets and improve the efficiency of the drug development system, for the benefit of both humans and canines.

\section{Financial \& competing interests disclosure}

The authors have no relevant affiliations or financial involvement with any organization or entity with a financial interest in or financial conflict with the subject matter or materials discussed in the manuscript. This includes employment, consultancies, honoraria, stock ownership or options, expert testimony, grants or patents received or pending, or royalties.

No writing assistance was utilized in the production of this manuscript.

\section{References}

1 DiMasi JA, Grabowski HG. Economics of new oncology drug development. J. Clin. Oncol. 25(2), 209-216 (2007).

2 Arrowsmith J. Trial watch: Phase II failures: 2008-2010. Nat. Rev. Drug Discov. 10(5), 328-329 (2011).

3 Bates SE, Amiri-Kordestani L, Giaccone G. Drug development: portals of discovery. Clin. Cancer Res. 18(1), 23-32 (2012).

4 Sharpless NE, DePinho RA. The mighty mouse: genetically engineered mouse models in cancer drug development. Nat. Rev. Drug Discov. 5(9), 741-754 (2006).

5 Cheon DJ, Orsulic S. Mouse models of cancer. Annu. Rev. Pathol. Mech. Dis. 6(1), 95-119 (2011).

6 Paoloni M, Khanna C. Translation of new cancer treatments from pet dogs to humans. Nat. Rev. Cancer 8(2), 147-156 (2008).

7 Shearin AL, Ostrander EA. Leading the way: canine models of genomics and disease. Dis. Model. Mech. 3(1-2), 27-34 (2010).

8 Fleming JM, Creevy KE, Promislow DE. Mortality in North American dogs from 1984 to 2004: an investigation into age-, size-, and breed-related causes of death. J. Vet. Intern. Med. 25(2), 187-198 (2011).

9 Thomas R, Seiser EL, Motsinger-Reif A et al. Refining tumor-associated aneuploidy through 'genomic recoding' of recurrent DNA copy number aberrations in 150 canine non-Hodgkin lymphomas. Leuk. Lymphoma 52(7), 1321-1335 (2011).

10 Angstadt AY, Thayanithy V, Subramanian S, Modiano JF, Breen M. A genome-wide approach to comparative oncology: high-resolution oligonucleotide aCGH of canine and human osteosarcoma pinpoints shared microaberrations. Cancer Genet. 205(11), 572-587 (2012).

11 Modiano JF, Breen M. Shared pathogenesis of human and canine tumors - an inextricable link between cancer and evolution. Cancer Ther. 6, 239-246 (2008).

12 Thomas R, Duke SE, Wang HJ et al. 'Putting our heads together': insights into genomic conservation between human and canine intracranial tumors. J. Neurooncol. 94(3), 333-349 (2009).

13 Lindblad-Toh K, Wade CM, Mikkelsen TS et al. Genome sequence, comparative analysis and haplotype structure of the domestic dog. Nature 438(7069), 803-819 (2005).

14 Davis RE, Ngo VN, Lenz G et al. Chronic active B-cell-receptor signalling in diffuse large B-cell lymphoma. Nature 463(7277), 88-92 (2010).

15 Honigberg LA, Smith AM, Sirisawad M et al. The Bruton tyrosine kinase inhibitor PCI-32765 blocks B-cell activation and is efficacious in models of autoimmune disease and B-cell malignancy. Proc. Natl Acad. Sci. USA 107(29), 13075-13080 (2010).

16 Advani RH, Buggy JJ, Sharman JP et al. Bruton tyrosine kinase inhibitor ibrutinib (PCI-32765) has significant activity in patients with relapsed/refractory B-cell malignancies. J. Clin. Oncol. 31(1), 88-94 (2013).

17 Sutter NB, Ostrander EA. Dog star rising: the canine genetic system. Nat. Rev. Genet. 5(12), 900-910 (2004).

18 Volk SW, Bohling MW. Comparative wound healing - are the small animal veterinarian's clinical patients an improved translational model for human wound healing research? Wound Repair Regen. 21(3), 372-381 (2013).

\section{Websites}

101 American Cancer Society. Cancer treatment and survivorship facts and figures 2012-2013 (2012). www.cancer.org/acs/groups/content/@ epidemiologysurveilance/documents/ document/acspc-033876.pdf

102 2011-2012 APPA National Pet Owners Survey. www.humaneresearch.org/content/20112012-appa-national-pet-owners-survey 